\title{
MUJERES: ENTRE LA AUTONOMÍA Y LA VIDA FAMILIAR
}

Women: between autonomy and family life

America Tonantzin Becerra Romeroํㄹ Paola Anayanci Santellan Palafox ${ }^{2}$

Fecha de recepción: 20 de junio de 2016

Fecha de aceptación: 27 de octubre de 2016

1- Nacionalidad: Mexicana. Grado: Doctorado en ciencias sociales. Especialización: Estudios culturales y de género. Adscripción: Universidad Autónoma de Nayarit. Correo: americabr01@gmail.com

2- Nacionalidad: Mexicana. Grado: Maestra en administración de negocios. Especialización: Estudios culturales y de género. Adscripción: Universidad Autónoma de Nayarit. Correo: paola.santellanp@gmail.com 


\section{Resumen}

La capacitación para el trabajo constituye una herramienta importante para las mujeres, ya que les permite estar en mejores condiciones para ingresar o mantenerse en el mundo laboral; sin embargo, ellas viven diversas tensiones al tratar de compaginar la vida familiar con la capacitación. Este texto es resultado de una investigación cualitativa basada en el método biográfico, diseñada para analizar el significado que tiene dicha capacitación en la vida personal, familiar y laboral de las mujeres. Entre los resultados se encontró, que el incremento en su formación no ha modificado sustancialmente la visión tradicional sobre las funciones sociales de las mujeres y los hombres, pero las ayuda a impulsar su autonomía personal y económica, sin renunciar a sus responsabiliades reproductivas y familiares. Las mujeres asignan valor tanto al ámbito doméstico como al público, y los consideran como espacios diferenciados que es necesario concertar.

Palabras clave: mujeres, capacitación para el trabajo, autonomía, vida familiar.

\section{Abstract}

Job training is an important tool in actual days for women, in order to be in better terms to enter or stay in the labor market; however, they suffer different experiences to combine job training and family life. This paper presents a qualitative research based on the biographical method, done with the purpose of analyzing the significance of job training in their personal development, family and working life. The results prove that the increase in the formation of women, has not substantially changed the traditional view about social roles of women and men, but promote their personal and economic autonomy, without sacrificing their reproductive and family responsabilities. Women give values to both domestically and public space, and they consider them as different areas that need to reconcile.

Keywords: women, job training, autonomy, family life. 


\section{Introducción}

diferencia de épocas anteriores, el actual mercado laboral demanda personas cada vez
más preparadas y calificadas. Esto ha provocado un incremento de establecimientos que
ofrecen capacitación para desempeñar alguna actividad económica, sobre todo a quienes no tiene acceso a la formación profesional en alguna institución de educación superior. En estos establecimientos que tienen la figura de institutos, centros de capacitación, academias o escuelas, puede observarse a una gran cantidad de mujeres que se adiestran en un oficio; por ejemplo, practican cortes de pelo, la elaboración de platillos o repostería, técnicas de relajación o de masaje, la creación de arreglos florales o de artesanías, la confección de vestidos o bordados, o aprenden inglés o computación, entre otras actividades. Es común ver además, a bebés en carriolas o niños que se entretienen mientras su mamá o hermana mayor salen de la clase. Gran parte de estas mujeres acuden con la expectativa de adquirir habilidades suficientes para conseguir un trabajo, mejorar su posición en él o crear su propia empresa, a fin de incrementar los ingresos familiares.

La búsqueda de espacios laborales por las mujeres aumentó notablemente desde las últimas décadas del siglo pasado, ante las recurrentes crisis que derivaron en una fuerte caída de la actividad económica, el incremento del desempleo formal, la reducción en el gasto público del Estado y en consecuencia, la acentuación de la pobreza y el traslado de la responsabilidad económica a las familias. En este escenario el hogar tradicional, en el que el hombre es quien aporta el sustento familiar, se transformó para dar paso a una dinámica distinta donde las mujeres juegan un papel fundamental como proveedoras de ingresos.

Pese al incremento de las mujeres en la actividad económica, en el mercado laboral persiste la inequidad de género debido a la división social del trabajo que permea en nuestra sociedad. Si bien se han ampliado los espacios laborales, existe un fenómeno de exclusión debido a que dichos espacios "no están disponibles para las mujeres, ya sea por problemas de calificación de la oferta, como por la definición social de las ocupaciones que son aptas para uno u otro sexo" (Aguirre, 2001; citado por Longo, 2009: 119).

La Encuesta Nacional de Ocupación y Empleo 2015 del INEGI, muestra que de las personas que integran la fuerza laboral en México, sólo el 38\% son mujeres, ganan 22\% menos que los hombres y más del $60 \%$ de su trabajo corresponde a actividades en el hogar no remuneradas.

De acuerdo con Longo (2009), la participación económica de las mujeres se caracteriza por situarse generalmente en el sector informal de la economía, registra altas tasas de desempleo y subempleo, se desempeñan en puestos socialmente desvalorizados por carecer de autoridad, son de tiempo parcial y con percepciones económicas inferiores. Silveira (2001) añade, que el acceso a los puestos vinculados a la toma de decisiones sigue restringido y les demanda a las mujeres ingentes esfuerzos de calificación, dedicación y postergación personal.

En general, las mujeres arriban al mundo laboral en condiciones de desventaja y con menores posibilidades de desarrollo, lo que aumenta su vulnerabilidad económica y social. Riquer y Tepichín (2001) plantean, que el incremento en la incorporación y permanencia de las mujeres en el sistema educativo, no ha sido condición suficiente para su participación en el mercado laboral en igualdad de condiciones respecto a los hombres. 
La división sexual del trabajo ha resistido el incremento en años de escolaridad de la población femenina y su participación en los mercados de trabajo.

Por lo tanto, la doble jornada laboral y doméstica, incluso para mujeres con alta escolaridad, sigue siendo la norma y no la excepción. (Riquer y Tepichín, 2001: 495)

Sin embargo, también se ha demostrado la estrecha relación entre la educación y el trabajo. Silveira (2001) señala, que "no hay dudas acerca del papel que el conocimiento desempeña en el desarrollo", de ahí que quienes tienen acceso a la educación, poseen mayores posibilidades de desarrollo personal y laboral.

En este contexto, la capacitación para el trabajo constituye una herramienta importante para las mujeres ya que les permite adquirir mayores conocimientos y habilidades, y estar en mejores condiciones para ingresar o mantenerse en el mundo laboral, aún cuando la incorporación a un instituto genere tensiones al tratar de conciliar las actividades del hogar con la capacitación. Por lo general, la formación de las mujeres conlleva diversas contradicciones personales que se originan al combinar el ámbito público con el familiar-conyugal.

Por lo tanto, los resultados no sólo depende de la oferta y desarrollo de los programas de capacitación, sino y sobre todo, de la experiencia vivida por las mujeres que acceden a ellos, de la forma en que incorporan el adiestramiento para el trabajo en su cotidianeidad y del significado que adquiere en su vida. Las necesidades, intereses y trayectorias vividas por las mujeres que recurren a la capacitación para el empleo o autoempleo son diversas, heterogéneas y complejas; en consecuencia, los alcances, beneficios y limitaciones son diferenciados.

Pieck (2012) afirma, que la indagación de la experiencia de las mujeres que se han capacitado para el trabajo permite revelar las percepciones, vivencias, logros, motivaciones y esfuerzos que las protagonistas nos dejan ver acerca de su vida y su contexto, y manifiestan la relevancia que ha tenido el hecho de haber aprendido una actividad específica.

Con base en lo anterior, se diseñó una investigación que permitiera ir más allá de un análisis utilitario de la capacitación para el trabajo a fin de estudiar el papel que juega en la vida personal, familiar y laboral de las mujeres, considerando las condiciones estructurales y culturales en que se desenvuelven. Los objetivos se centraron en el análisis de dos aspectos básicos: a) los significados que adquiere la capacitación para el trabajo en la vida de las mujeres, y b) las formas en que concilian la vida familiar con la capacitación que reciben.

La indagación se realizó en la ciudad de Tepic, capital de Nayarit, estado donde la transversalidad de la perspectiva de género apenas comienza (Guzmán, Rodríguez y González, 2008). A pesar de que la igualdad de género se encuentra estipulada en la carta magna nayarita como un valor primordial de los derechos de mujeres y hombres en el estado, se hacen evidentes prácticas diferenciadas entre la igualdad de hecho y de derecho (Cervantes, 2013). Cabe señalar que en el 2005 y de acuerdo con Programa de las Naciones Unidas para el Desarrollo (PNUD), la entidad ocupó el lugar número 23 en el conjunto de entidades del país respecto al índice de desarrollo relativo al género (IDG), valor que sintetiza las desigualdades entre mujeres y hombres en aspectos como vida saludable, tasa de alfabetización de adultos y nivel de vida decoroso según los ingresos percibidos.

Aunque existe un incremento notable en las investigaciones sobre la participación económica de la mujer, estas atienden sobre todo aspectos de la relación mujer-empleo-empresa. Dichos estudios en el 
contexto nayarita son escasos, aún más los que hacen referencia a la relación mujer-capacitación para el trabajo-empleo.

\section{El sistema sexo-género}

Los contextos socioculturales en los que se insertan las mujeres al mundo laboral pueden analizarse desde la perspectiva del sistema sexo-género, que hace referencia a las formas de relación establecidas entre mujeres y hombres dentro de una sociedad específica, bajo un sistema de poder que propicia condiciones sociales desiguales para mujeres y varones, en función de los papeles y actividades que les han sido asignadas socialmente, y donde lo femenino queda subordinado a lo masculino (Aguilar, 2008).

Desde esta perspectiva es posible observar que en el mundo laboral la división del trabajo queda supeditada a la diferencia sexual, más que a las capacidades personales. En la sociedad mexicana a las mujeres se les atribuyen las funciones de reproducción y el trabajo de cuidados, actividades no remuneradas y sin reconocimiento social; es decir, el ámbito de lo privado. Mientras que a los hombres se les designan las actividades de producción valoradas y remuneradas, con poder, autoridad y estatus: el ámbito de lo público. De acuerdo con el Instituto Nacional de las Mujeres (2004), el ámbito privado hace referencia al espacio y prácticas relacionadas con el hogar, la familia, el trabajo y cuidado doméstico; en contraste, el ámbito público se conforma por el espacio y prácticas relacionadas con la producción y la política, y en él se constituyen las estructuras socioeconómicas de una sociedad.

Silveira plantea que durante siglos, a los varones les corresponde el trabajo productivo y a las mujeres el casamiento y la maternidad, o sea el trabajo reproductivo, "sólo que el primer tipo de proyecto ha llevado a la independencia económica y al pleno reconocimiento ciudadano y el segundo, a la dependencia y a una ciudadanía delegada." (Silveira, 2001: 459)

Estas condiciones son resultado de las políticas sociales y económicas existentes, pero a la vez se enraizan en la cultura y formas de pensar y actuar respecto a lo que es una mujer y un hombre en nuestra sociedad. Colín, Gallaga y Martínez (2011) afirman, que a pesar de que las mujeres tienen mejores niveles educativos y su participación económica va en crecimiento, aún son presas de aspectos ideológicos como el "techo de cristal", que son barreras invisibles que enfrentan conforme avanzan laboralmente, y el "suelo pegajoso" que representa la adherencia de las mujeres al trabajo de cuidados, trabajo doméstico, y responsabilidades afectivas que limitan su desarrollo personal, profesional y laboral.

De este modo la sociedad mantiene una ideología tradicional que propicia prácticas de discriminación, segregación y hostigamiento sexual entre otras, donde las mujeres resultan ser las más perjudicadas (Instituto Nacional de las Mujeres, 2003).

Las mujeres se enfrentan a una diversidad de dificultades y obstáculos que no pueden ser explicados solamente por la dinámica del mercado de trabajo, sino que además existen otras dimensiones socioculturales, como las relaciones de poder, representaciones sociales del trabajo, roles (el "deber hacer" y "deber saber") y estereotipos de género (el “deber ser” y "deber sentir”) que regulan las prácticas sociales (Longo, 2009).

Por ello se hace imprescindible analizar estas situaciones desde la perspectiva de género, ya que implica ampliar el horizonte para tratar de comprender la problemática de las relaciones que sostienen mujeres y hombres en la sociedad actual (Lamas, 1996). 
Además, considerar la perspectiva de las propias mujeres toma relevancia frente a la tendencia de la ciencia social tradicional que basa sus análisis en la visión masculina y, como plantea Harding (1987), no es suficiente con sumar o agregar a las mujeres como sujetos de estudio, sino que las problemáticas deben partir de las experiencias de las mujeres, las cuales constituyen un indicador significativo de la "realidad".

\section{Capacitación para el trabajo y perspectiva de género}

Como categoría analítica, el género es un constructo sociocultural que analiza las relaciones entre mujeres y hombres a partir de su diferencia sexual, y cuestiona la desigualdad y discriminación entre los sexos basada en el "deber ser" y "deber hacer" de mujeres y hombres. Silveira explica que el concepto de "género" hace referencia

... a la asignación social diferenciada de responsabilidades y roles a hombres y mujeres que condiciona el desarrollo de sus identidades como personas, de sus cosmovisiones y de sus proyectos de vida. Esta asignación está basada en las pautas culturales, hábitos y condicionamientos sociales vigentes - estereotipos sociales- que definen y valoran roles y tareas de acuerdo al sexo, reservando prioritariamente para el hombre la esfera pública de la producción y para la mujer la esfera privada de la reproducción y el cuidado de los otros. Se hallan presentes desde el inicio mismo del proceso de socialización y son transmitidos desde el hogar, confirmados en la escuela y expandidos a través de los medios de comunicación masivos. (Silveira, 2001: 458)

Lo anterior implica que las tareas, actitudes y formas de pensar derivadas del género, corresponden a aprendizajes que se construyen en forma social y en contextos histórico-sociales específicos.

De acuerdo con Lamas, la perspectiva de género implica reconocer que una cosa es la diferencia sexual y otra cosa son las atribuciones, ideas, representaciones y prescripciones sociales que se construyen tomando como referencia a esa diferencia sexual (Lamas, 1996).

Gamba (2008) agrega, que esta perspectiva expone las relaciones de poder que se dan entre los géneros (en general favorables a los varones y discriminatorias para las mujeres), las cuales atraviesan todo el entramado social y se articulan con otras relaciones sociales como las de clase, etnia, edad, preferencia sexual y religión.

El género se constituye, por ende, en un instrumento crítico de análisis y es una variable socioeconómica de base sobre la que influyen las otras variables generadores de diferencias por lo que los logros alcanzados en equidad de género encuentran sus frenos en la supervivencia de otras inequidades y viceversa (Silveira, 2001: 459).

En el mundo laboral la perspectiva de género supone analizar cómo se organizan y distribuyen los tiempos y los espacios tanto en el hogar como en el trabajo, así como las creencias e ideologías más profundas con respecto al trabajo femenino y masculino. Además, contribuye a identificar cómo las tradiciones y 
costumbres culturales limitan la participación económica de las mujeres en la esfera pública (Lamas, 1986).

La investigación científica realizada desde esta visión, ha mostrado la prolongada situación de marginación de las mujeres, la desvalorización de la mano de obra femenina tanto en el ámbito laboral como en el familiar, las causas de su discontinua participación en el mercado de trabajo, la calidad y tipo de formación y capacitación que éstas reciben, la introyección de un modelo de mujer basado en la maternidad y el cuidado, así como la posición de subordinación que mantienen con respecto a los hombres.

En el caso de la capacitación para el trabajo, la perspectiva de género permite dar cuenta de las implicaciones que tiene dicha capacitación en la vida de las mujeres ya que la salida del hogar trae aparejada para ellas, la necesidad de compatibilizar "sus responsabilidades" en las esferas productiva y reproductiva.

Es en el ámbito familiar donde la división sexual del trabajo se acentúa en todo su esplendor. En él se reproducen y refuerzan las ideologías y prejuicios más arraigados con respecto a los comportamientos y funciones que se espera se desempeñen socialmente, según se pertenezca a uno u otro sexo.

Mujeres y hombres no compiten en el mismo mercado de trabajo, sino que existen dos sectores claramente diferenciados, uno de trabajo femenino y otro masculino, cada uno con requerimientos específicos sustentados en la diferencia sexual. Esto repercute en el tipo de formación y capacitación para el trabajo que se ofrece a mujeres y hombres.

Riquer y Tepichín explican que una parte de las demandas feministas se dirige a reivindicar el derecho que la mujer tiene a trabajar por un ingreso que les daría "la autonomía necesaria para enfrentar y modificar valores, normas y prácticas que han impedido u obstaculizado su entrada a las actividades centrales de la era moderna: ciencia, política y economía." (Riquer y Tepichín, 2001: 494)

Asimismo, las autoras anteriores indican que aunque se ha puesto en debate la relación entre escuela y trabajo, la situación de las mujeres queda relegada; por lo que, el sexo constituye sobre todo, una variable más, pero no un factor explicativo. "El debate feminista, académico y militante, que plantea el incremento en la incorporación y permanencia de las mujeres en la escuela y en los mercados de trabajo, no agota ni ha resuelto la problemática de la desigualdad de género." (Riquer y Tepichín, 2001: 496)

En esta investigación, el análisis de la capacitación para el trabajo desde la perspectiva de género permitió identificar factores históricos-estructurales provenientes de la dimensión de género. Además, el escrutinio de la experiencia de capacitación de las mujeres, propició un mayor entendimiento de los obstáculos que enfrentan y de las oportunidades que se les presentan, así como de los recursos y estrategias que se hacen necesarios para contribuir a su crecimiento y desarrollo tanto personal como profesional.

\section{Conciliación entre capacitación para el trabajo y vida familiar}

Uno de los principales aspectos que inciden en las oportunidades y obstáculos de la capacitación para el trabajo de las mujeres, es lograr la conciliación entre las exigencias vinculadas con su formación y su rol como madres, esposas o hijas. En las últimas décadas, la estructura familiar ha mostrado una disminución de hogares nucleares y el aumento de hogares conformados por la madre y sus hijos. Asimismo, la familia típica donde la madre se dedica exclusivamente a las labores domésticas y el padre es el único proveedor, se difumina debido a que la insuficiencia económica hace indispensable la aportación monetaria de las mujeres. 
Sin embargo, el ingreso de las mujeres al espacio público genera controversias ya que transforma la dinámica tradicional de los hogares, por lo que conciliar la vida laboral y familiar constituye un desafío para las mujeres dentro de la sociedad mexicana.

La Fundación Mujeres conceptualiza la conciliación de la vida laboral y familiar como:

La estrategia por la que se introducen sistemas de permiso por razones familiares, de atención a la infancia y a personas de edad avanzada, y creación de una estructura y organización del entorno laboral, que facilite a hombres y a mujeres la combinación del trabajo y de las responsabilidades familiares (Fundación Mujeres, 2007: 7).

En esta conciliación además de los espacios público y privado, algunos autores integran al ámbito doméstico por estar relacionado directamente con la vida familiar. De acuerdo con Murillo el espacio privado queda asignado al desarrollo de la individualidad, es el lugar del disfrute de quienes pueden desentenderse de las obligaciones. En tanto, el ámbito doméstico es donde tiene lugar la reproducción, el trabajo de cuidados y la satisfacción de las necesidades básicas del núcleo familiar. Este último no le pertenece a la mujer, sino que se encuentra en función de los otros (Murillo, 1996; Luque, 2012).

El ámbito doméstico no constituye el espacio donde las mujeres puedan destinar tiempo para sí mismas, "libres" de las obligaciones del hogar que les permitan construir un tiempo propio para su persona. Sino por el contrario, es un espacio de relaciones que lo dedican a los otros, donde el tiempo en lugar de detenerse, avanza sin freno; en cambio, para los hombres representa el lugar para disfrutar de su tiempo libre, dedicado para sí mismos, alejados de las responsabilidades laborales.

Con base en lo anterior, el ingreso de las mujeres al mundo laboral entra en juego no sólo con las dinámicas familiares sino también con el desarrollo personal. Como Reyes (2005), en el proceso de realización de las mujeres prevalecen contradicciones personales que se originan en la vida "social-pública" y la vida "privada-familiar", así como acciones y prácticas sociales e institucionales que complican dicho proceso. Las maneras de vincular las relaciones en el ámbito "familiar-conyugal" es uno de los límites sociales externos que más ha impactado en la vida de las mujeres.

Las situaciones que ellas enfrentan al entrar en el mundo laboral, se reproducen de manera automática cuando buscan espacios de capacitación que pueden propiciar oportunidades laborales o ingresos económicos. Desde la visión tradicional, las tensiones familiares derivadas de esta situación constituyen un problema de las mujeres y deberán ser ellas quienes lo solucionen; por lo tanto, se ven obligadas a buscar estrategias para acceder a la capacitación y lograr una participación económica activa dentro del mercado de trabajo.

De acuerdo con Sifuentes (2002), la incorporación de las mujeres al mercado de trabajo no significa su deslinde de las responsabilidades del hogar; por el contrario, crea la doble o triple jornada para mujeres que participan en actividades económicas y se encargan de las labores domésticas y de la familia, y a la par, continúan su educación (Tunal, 2007; citado por Rocha y Cruz, 2013).

Las aportaciones teóricas citadas, permiteron tener un referente conceptual, contextual y metodológico, a partir del cual se diseñó el trabajo de campo de la investigación. 


\section{Diseño metodológico}

Con el fin de analizar los significados que las mujeres le atribuyen a la capacitación para el trabajo, se realizó la investigación desde el enfoque cualitativo, transversal y no parametral. Asimismo, se recurrió al método fenomenológico para rescatar la experiencia de las mujeres "tal como es vivida y percibida por el sujeto" (Martínez, 2004). De acuerdo con este método, el investigador debe reducir la influencia de sus propias ideas y teorías previas, y hacer un esfuerzo por captar la realidad que se presenta de manera vivencial, para dar relevancia al "mundo vivido" por las personas. Esto se complementó con el método biográfico como medio para explorar en forma holística la experiencia vivida; en específico, se empleó el método de relatos biográficos múltiples y paralelos que hacen referencia a diversas trayectorias de vida que han transcurrido sin que necesariamente converjan o generen vínculos entre sí (Sanz, 2005).

Para realizar la investigación se construyeron dos categorías de análisis, en concordancia con los objetivos de la investigación: el significado de la capacitación para el trabajo y la conciliación de la vida familiar con dicha capacitación. La primer categoría hizo referencia a los procesos de formación para el trabajo que han tenido las mujeres, e incluyó indicadores como los motivos para capacitarse, expectativas planteadas, estrategias aplicadas, limitantes encontrados y logros obtenidos. La segunda, abordó las maneras de combinar las responsabilidades familiares y del hogar con el proceso de capacitación para el trabajo; entre sus indicadores se tuvieron los roles en la familia, las responsabilidades y decisiones familiares, la gestión del tiempo y las acciones realizadas para combinar la familia y la capacitación.

El trabajo de campo permitió identificar más de una decena de instituciones de capacitación para el trabajo en Tepic, que se pueden clasificar en tres tipos: públicos, privados y sociales. Debido a la diversidad de establecimientos, se seleccionaron a los más reconocidos y demandados de cada tipo: de los públicos se eligió al Instituto de Capacitación para el Trabajo del Estado de Nayarit, de los privados a la Escuela de Belleza "Rosalva", y de los sociales a la Academia Dominical "Santa Teresita del Niño Jesús". Una vez seleccionados, se visitaron las instalaciones, se presentó el proyecto a las autoridades respectivas y se pidió información general como antecedentes, público al que va dirigido, cartera de cursos, duración, costos y horarios.

Para tener acceso a las mujeres sujeto de estudio se recurrió dos estrategias. La primera consistió en inscribirse a determinados cursos de capacitación con la finalidad de tener cercanía con las estudiantes, conocerlas y convivir con ellas dentro del entorno de la propia capacitación; con el tiempo se les comentó sobre el proyecto de investigación y, gracias a la confianza creada, accedieron a compartir sus experiencias y emociones. De esta manera se entrevistaron de manera voluntaria a 18 mujeres de los centros de carácter público y social. No obstante, la estrategia no pudo aplicarse en la institución privada debido a la larga duración de los cursos y su elevado costo, por lo tanto se recurrió a las autoridades de dicho centro para solicitar su apoyo y extender la invitación a las estudiantes a colaborar en la investigación, a la que respondieron seis mujeres. Aunque con esta estrategia la participación de las estudiantes fue más formal que en la primera, la información que proporcionaron fue suficiente para cubrir los indicadores y las categorías de análisis establecidas.

La muestra se tomó con el criterio de saturación propuesto por Guest, Bunce y Jonhson (2006), quienes lo plantearon como el punto en el que cual no se observa nueva información o temas en los datos. En el caso de investigaciones basadas en entrevistas, el número de testimonios depende del tema de estudio, 
los hallazgos que se pretenden encontrar, la complejidad de las categorías de análisis y la credibilidad de los datos obtenidos.

Por lo tanto, la muestra no se definió desde el inicio sino que se fue conformando con las mujeres que aceptaron compartir su experiencia al capacitarse para el trabajo. Tampoco fueron determinantes factores como el nivel socioeconómico, edad, estado civil o número de descendientes, ya que se buscó que la muestra fuera lo más diversa posible para obtener mayor información y variedad de vivencias.

Como instrumento para la recolección de los testimonios se empleó la entrevista focalizada de corte biográfico, estructurada bajo ejes temáticos. Una vez recabados los datos, se realizó su procesamiento con el apoyo del programa Atlas.ti, a partir de lo cual se integraron los resultados con base en la estrategia interpretativa de datos cualitativos propuesta por Miles y Huberman (1994), que abarca tres etapas: reducción de datos, disposición y transformación de datos, y obtención de conclusiones.

Los resultados se presentan de acuerdo con las categorías de análisis planteadas en el diseño de la investigación; en ellos se insertan, a manera de ejemplo, parte de los testimonios expresados por las propias entrevistadas.

\section{De qué mujeres y qué capacitación hablamos}

En total se entrevistaron a 24 mujeres, que pertenecen al nivel socioeconómico medio bajo. Su edad varía ampliamente desde los 15 hasta los 54 años. Asimismo, su estado civil es diverso: 13 solteras, seis casadas, dos separadas, una divorciada y dos en unión libre. El grado educativo también fue heterogéneo: 10 con educación básica secundaria, cuatro con carrera comercial, dos con educación media superior, tres con licenciatura inconclusa y cinco con licenciatura terminada. Esto pone en evidencia que no existe una edad, un estado civil o un grado educativo específico para que las mujeres acudan a la capacitación para el trabajo.

A través de la indagación se observó que con excepción de dos personas, el resto de las mujeres han ingresado a varios programas de capacitación, de manera que se contabilizaron un total de 86 cursos en los cuales han participado. La oferta de cursos puede cambiar cada semestre según la disponibilidad de instructores e infraestructura, pero en general se mantiene una amplia variedad por lo que es posible encontrar un listado de más de 40 cursos en una sola institución. A pesar de esta diversidad, la mayoría de las mujeres buscan tres temas principalmente. Los más populares, el 26\%, están vinculados con la belleza como cultora de belleza, estilismo, maquillaje y decoración de uñas; esto se debe en parte, a que pueden utilizar lo aprendido en la propia persona, pero también a los estereotipos de género asimilados desde la infancia y socializados en la familia. Como indicó una de las entrevistadas:

Porque siempre me ha gustado desde chiquita el maquillaje, que traer bien arreglado mi cabello, que peinar a mis Barbies. Desde chiquita entonces así era, entonces como tengo una tía que estudió cultura de belleza, entonces como que ella me metió el gusanito de estudiar eso. (Alisson)

En segundo lugar, el $21 \%$, se refiere a cursos sobre masajes y temas semejantes como reflexología, biomagnetismo y flores de Bach. El propósito de tomar estos programas generalmente se relacionó con la posibilidad de ayudar a miembros de la familia que requerían terapias específicas. 
El tercer lugar, el 15\%, lo ocupan los cursos relacionados con la preparación de alimentos como cocina, repostería, mesa de postres, pasteles, fondant y pequeñas industrias.

La suma de estos tres grupos asciende al 62\%. El resto de los cursos se puede clasificar de la siguiente manera: el $9 \%$ están vinculados a corte y confección, tejidos y bordados; el $8 \%$ a manualidades, velas, arte floral y fieltro; $6 \%$ a la atención a la salud como enfermería y primeros auxilios, y $6 \%$ a secretaria ejecutiva, capturista de datos y francés. El resto de los cursos son de carpintería, electricidad, mecánica y tapicería.

La selección de los programas depende por una parte, de la oferta de las instituciones de capacitación la cual contiene un alto porcentaje de cursos relacionados con el ámbito femenino; y por otra, obedece a la elección de las mujeres. Si bien dicha elección se relaciona con la posibilidad de aplicar los conocimientos en el entorno cercano como la persona y la familia, también se basa en la perspectiva que tienen de sí mismas, como mujeres y de su desarrollo.

La información obtenida pone de manifiesto que en la oferta y la selección de los cursos, permea la visión tradicional de la mujer y los estereotipos de género que asignan a las mujeres los espacios vinculados a la belleza, la salud y la atención o cuidado de la familia.

\section{El significado de la capacitación para el trabajo}

La capacitación para el trabajo pudiera imaginarse como una fórmula simple: se ingresa a un instituto, se recibe la habilitación y se pone en práctica lo aprendido. Sin embargo, dista mucho de ser así ya que es un proceso complejo, diverso y con múltiples condicionantes. La investigación realizada permitió observar que este proceso puede derivar en diferentes trayectorias y distintos resultados, gracias al significado que adquiere esta capacitación en la vida de cada persona.

Existen diversos motivos que llevan a las mujeres a ingresar a un programa de capacitación: socializar, entretenerse, como terapia ocupacional o practicar un oficio. Sin embargo, de tras de todo ello está una razón fundamental: el deseo de aprender, que se basa a su vez, en el reconocimiento de la falta de habilidad para realizar una actividad específica. Otra de las entrevistadas, quien tenía conocimientos básicos de costura y decidió ingresar a un curso de corte y confección, comentó:

Entonces yo, realmente no me sentía capacitada para hacer ciertas tareas. Entonces yo dije "no, a mí me falta aprender más”, más práctica en las máquinas, porque eran máquinas industriales, porque yo nunca había manejado máquina industrial, sólo que la aguja y eso. (Isabel)

Este aprendizaje se transforma en una herramienta importante cuando está ligado a la posibilidad de incrementar los ingresos económicos. En la indagación se puedo detectar a mujeres que tuvieron la oportunidad de tener un mejor puesto de trabajo (como pasar de encargadas de limpieza a secretaria o a asistente en una clínica de belleza), pero lo rechazaron al carecer de la habilitación respectiva y decidieron iniciar su capacitación. Pero también se encontraron aquellas que buscaron la capacitación como vía directa para resolver las necesidades básicas de la familia. En este caso se encontraron varias de las mujeres que se abordaron en la investigación, ante las limitaciones de cubrir los aspectos básicos (como la alimentación) o los “antojos" propios o de los hijos. 
... fue cuando entonces decidí yo capacitarme, para poder aportar yo también. (Sonia)

Margarita tiene 40 años estudiando en la academia "Santa Teresita", uno de los cursos que tomó fue el de cocina. Ella relató la manera en que tuvo que preparar pan de platano y venderlo para sacar a delante a su familia.

Mi esposo ese día, este no le pagaron, los ingenieros a veces no les pagan. Entonces mi hija estaba en la secundaria, ya estaba para salir de tercero y llevaba el primer lugar de la escuela. Entonces no tenía para pagar la escuela de mi hija, y ya nos pedían... Entonces, dije: "Bueno, ¿qué hago?". Ese día me quedé: "Pues tantas cosas que sé, no me voy a quedar sin comer". Dije yo: "Bueno". Ese día nos regalaron tantos plátanos... Mi papá vive en el rancho, en la playa. ¿¿Qué hago con tanto plátano?... ha! pos el pan de plátano". (Margarita)

Un caso semejante es el de Martha, quien hace años tomó un curso para secretaria ejecutiva pero sabe que ahora sus conocimientos están desactualizados, que su situación ha cambiado y que existen condicionantes de género en el empleo:

Yo me veo en la necesidad de trabajar para tarer más dinero a mi casa, porque lo de él [su pareja] no alcanza, no le aumentaban y hasta la fecha me sigue dando lo mismo. Pero me doy cuenta que ya no me dan trabajo, porque a las mujeres nos quieren hasta los 35 años, solteras y modelos, bien hechas, bien formaditas, ¿edá? Entonces yo digo: "ya no me van a dar trabajo". Entonces yo, entre que estudié y trataba de conseguirme un trabajo y ganar mejor, se van pasando los años, los niños van creciendo, tienen necesidades y entonces yo veo la necesidad de seguir trabajando... y siempre te encontrabas con que la experincia... Entonces digo: "necesito poner un negocio". Entonces digo: "me gustaría estudiar cultura de belleza", todo el mundo se corta el cabello, ¿edá?.. Entonces decido estudiar cultura de belleza. (Martha)

La práctica del oficio aprendido comienza en entornos cercanos y conocidos. Muchas veces inicia con la propia familia como dar masajes a la mamá, los hermanos, los hijos o a la pareja si están cansados; ayudarlos en las terapias de recuperación cuando sufren una fractura o lesión; prepararles platillos o postres especiales; cortarles el pelo, peinar y maquillar a las hermanas o a la mamá para eventos exclusivos, o hacerles prendas de vestir cuando lo requieren. Por lo general, esta práctica se va extendiendo a otros entornos como la colonia, los vecinos o amigos a quienes cobran precios módicos, y termina con el público en general, con costos más altos. Los ingresos económicos obtenidos no sólo se disponen para la economía familiar, sino también para mejorar su trabajo: mayores insumos, mobiliario, renta de un local propio o incluso, la contratación de ayudantes.

Sentir que su trabajo es valorado, reconocido e incluso demandado por diferentes personas, les permite a las mujeres salir del ámbito familiar y explorar nuevos entornos. Este proceso, refleja el grado de confianza que van adquiriendo no sólo en el oficio que practican, sino en sí mismas, ya que implica asumir una nueva actividad y un nuevo rol en el hogar como proveedoras de ingresos. Fue común encontrar expresiones como "me dio seguridad", "me animé", "agarras más confianza", "encontrarme, enfrentarme a los miedos", "darme cuenta lo que me falta o en lo que debo trabajar", "se me fue retirando la timidez", "me ha enriquecido emocionalmente", "me siento más agusto", “mejoré como persona", "me siento orgullosa porque lo logré”. 
Estos cambios se apoyaron en parte, en las relaciones sociales generadas durante la capacitación.

Fue un poquito difícil, a veces estuve a punto de ya no ir. Pero pues una persona me apoyó y dije: "ino!, tengo que sacarlo... y ya con mis compañeras. Hubo una maestra con la que me conecté mucho y me decía: “no, no, Sonia, no dejes, no tires lo que ya llevas ganado, si te entiendo, pero ya llevas mucho, no lo dejes"... Te ayudan mucho, a veces te dan lecciones, te hacen ver las cosas de diferente manera. [Me siento] más segura, porque ya tenía más conocimientos, podía ofrecer diferentes masajes y sobre todo, con más fortaleza. Capacitándote en algo que te gusta y estar conviviendo con diferentes personas, porque a veces ves situaciones más difíciles que la tuya, y dices: "sí puedo, puedo seguir adelante"... Entonces, sí fue lo que creo que sentí: más seguridad, más fortaleza y entera. (Sonia)

No obstante los impedimientos que puedan encontrar, las mujeres que deciden culminar su capacitación, describen su formación como muy satisfactoria. Por ello, es frecuente que al concluir un curso, inician otro, a veces con el fin de complementar lo que han aprendido; por ejemplo, llevan secuencia de masajes, estilismo y reflexología, o capaturista de datos, secretaria ejecutiva y pequeñas industrias. Sin embargo, en la mayoría de los casos, la intención es continuar con la capacitación, de manera que se pudo encontrar mujeres hasta con 17 cursos en diversos centros o institutos de capacitación.

Hay gente que me dice: "o sabe hacer todo y no le pega a nada". No es cierto eso, es mentira. ¡Qué padre que una mujer sepa hacer de todo!, porque de cada detalle, le saca jugo. No seremos millonarias, no sabemos todo, pero no se me atora nada". (Margarita)

Varias entrevistadas señalaron que esta experiencia las hace sentir "más enteras", "ser una mujer completa o total", "me siento una mujer bien realizada, bien hecha". En ocasiones, este sentimiento se genera al observar los logros realizados por la pareja y las carencias identificadas en el desarrollo propio, como comentó Aída (hablándo de sí misma en tercera persona):

Bueno, a Aída la veo como que más, un poco más segura porque se sentía insegura por respecto a que mi esposo ya era licenciado y yo nada, nada tenía. Y dije: “cómo es posible?, él licenciado y yo nada ¡no!, no me lo voy a permitir", porque sí se siente uno mal, bueno al menos en mi persona [...] Me sentía mal en el sentido de que, me sentía no realizada, no complementada con esa parte de mí. (Aída)

Los nuevos aprendizajes, las relaciones sociales establecidas, sentirse mejor posicionadas en el mundo laboral, incrementar el ingreso familiar y darse cuenta que pueden salir adelante incluso en condiciones desfavorables, les permitió ampliar la visión que tenían respecto a sus posibiliades de desarrollo. Para algunas mujeres la confianza adquirida les hizo reflexionar en nuevas expectativas a futuro o incluso, retomar sueños o deseos postergados.

Yo decía: "cuando mis hijos estén grandes y se casen, yo quiero irme de misionera", yo eso decía. Yo sé que es bien difícil, que a lo mejor, no sé, es bien difícil; pero apenas, a mis cincuenta y dos años, casi ciencuentra $y$ tres, a lo mejor voy a empezar. (Isabel) 
Como se puede observar, para las mujeres el sentido de la capacitación no se relaciona solamente con los ingresos que generan a partir de ella, sino que tiene que ver con la autonomía económica y personal. Tal como ha planteado Silveira (2001), más allá de la heterogeneidad, las mujeres comparten la preocupación por la afirmación de la autonomía, y la formación y el trabajo son factores imprescindibles para conquistarla.

La autonomía económica implica la posibilidad de tomar decisiones de la disposición de los ingresos de manera independiente; en caso contrario, esta decisión queda supeditada a quien aporta dichos ingresos, como el esposo o la pareja, o los padres. Esto constituye de alguna manera, espacios de libertad aunque sea relativa.

Ah, pues para mí significa mucho porque puedo darme mis gustos, comprarme lo que yo necesito sin tener que pedir, este, comprarle algún gustito a mis hijos, invitarlos al cine o a comer. (Aída)

La práctica del oficio y la autonomía adquirida les permite aumentar su participación en el ámbito público y el ámbito doméstico y la actividad de ama de casa se resignifican. De ahí que la capacitación y la formación profesional en general, se concibe como una pauta para lograr el bienestar familiar y un indicador de calidad de vida. Para las mujeres solteras, conlleva la posibilidad de planear una vida independiente a futuro.

... cuando creciera y si Dios me lo permite, tener una familia, y con algo solventarla, o sea tener un algo. (Leonela)

En el caso de las mujeres que han conformado un hogar, es una oportunidad para retomar su desarrollo personal. Además, tratan de inculcar la capacitación como un valor en los hijos, pero sobre todo, en las hijas.

... con esa superación, enseñamos a nuestras hijas. (Margarita)

Todo esto es siempre pensando en ellos, en mis hijos. Yo creo que estoy sembrando una semillita en ellos, quizá ahora no lo ven, pero siento que sí estoy sembrando una semillita y estoy poniendo el ejemplo de la lucha, de la constancia, del aprendizaje y que este, no hay edad para aprender. (Martha)

Esto pone en evidencia que las mujeres tienen conciencia de las dificultades que presenta el mercado laboral, así como de la desigualdad de oportunidades para mujeres y hombres, ante las cuales buscan alternativas de éxito no sólo para sí mismas sino para la familia, lo cual constituye al final de cuentas, la principal motivación para buscar la capacitación para el trabajo.

\section{Entre la vida familiar y la capacitación para el trabajo}

Como se mencionó, las mujeres que entran a programas de capacitación para el trabajo buscan en gran parte, encontrar un medio para generar ingresos económicos. Sin embargo, este proceso conlleva 
un reacomodo en su vida cotidiana y en las responsabilidades que tienen en el hogar. Las mujeres solteras expusieron estar ligadas a actividades domésticas como la comida, el aseo y ordenamiento de la casa, la atención a los padres y hermanos; en tanto, las que han formado una familia propia, tienen además la responsabilidad de los hijos. Aunque no estén reconocidas de manera formal, estas actividades constituyen una jornada laboral, a la cual no renuncian aunque ingresen a la capacitación.

... no se me hacía justo que él [su pareja] tuviera absolutamente toda la responsabilidad. Sí, yo me hacía cargo de la casa, de los niños, pero aún así yo pensaba: es que yo puedo ayudarlo, yo puedo apoyarlo. (Sonia)

La conciliación que ellas han realizado entre el hogar y su formación e ingreso al mercado laboral, implica el arreglo de un conjunto de variadas situaciones y emociones, como se explica a continuación:

a) Ajuste de horarios y recursos económicos para la formación. Las mujeres buscan la opción menos costosa para no afectar los recursos del hogar, de ahí que las instituciones de capacitación para el trabajo públicos y sociales tengan mayor demanda. Además, prefieren los programas que se ofrecen los domingos para continuar con la atención semanal de la familia y la casa.

b) Mis hijos son primero. Las mujeres postergan la capacitación y entrada al mercado laboral, por dar prioridad a la atención de su familia. Incluso algunas entrevistadas mostraron sentimiento de culpa por dejar a los hijos al cuidado de otra persona, como Aída, quien trató de extender su jornada de trabajo para incrementar sus ingresos.

... lo dejé hasta las ocho de la noche y dije: "o sea no, cómo voy a tener a mi hijo tan tarde por ganar un peso más". Fue cuando dije: "prefiero ganar si quieres lo mínimo, pero estar con mi hijo chiquito". Porque ese día me sentí tan mal, que lo llevé, me acuerdo, que a cenar un helado y unas cosas que le fascinan, y dije: "no vuelvo a llegar por ti tan tarde, hijo". (Aída)

Además, la atención a los hijos debe ser resuelto por las propias mujeres; en algunos casos buscaron el apoyo de la pareja u otros miembros de la familia, en otros, ellas siguieron a cargo.

A mis niñas, siempre las cargué para todos lados, nunca las dejé encargadas (Margarita)

Lo primero que me surgió era, ¿dónde voy a dejar a mi hijo?, especialmente el más chico. Y fue cuando ya empecé yo a platicar con él y veía la forma en que, pues pudiera acompañarme. Entonces me esperaba afuera, y yo con el pendiente acá. (Sonia)

c) Las mujeres tienen que asegurar el orden de la vida doméstica. En general, la familia acepta que las mujeres ingresen a la capacitación para el trabajo, pero les delegan a ellas la resposabilidad de reorganizar la vida doméstica. Como resultado, ingresan a procesos de capacitación pero no renuncian a su trabajo de cuidado y reproducción; 
como indican Riquer y Tepichín (2001), enfrentan la difícil compaginación entre el ciclo laboral y la vida familiar. Es decir, las mujeres asumen que la reorganización de las actividades domésticas es una responsabilidad propia, aunque los beneficios se extiendan a la familia.

Temprano tenía que organizarme todo. Ya tenía su lonche y todo, comida, todo listo; hasta dejaba mi quehar hecho. Hasta el día de hoy me acuesto muy noche y hago lo posible de tener todo al margen. (Margarita)

Las mujeres crean redes de apoyo con integrantes de la familia y amistades, pero se asumen como principales responsables. Son pocos los testimonios donde los miembros varones de la familia (la pareja, padres o hermanos) las sustituyen; por lo general, ellos continuan con su vida cotidiana sin mayores alteraciones. Con los hijos e hijas es diferente ya que las mujeres les delegan tareas domésticas según la edad, de manera que aumentan progresivamente, y negocian con ellos y ellas los tiempos de convivencia y apoyo.

Él [su pareja] está encantado. Pero pues también dice que lo piense, lo piense más bien yo, porque pues quien lleva más la carga es uno, ¿verdad? Ellos qué, nomás se van... y sí podría convivir un poco más con la criatura, pero quien va a estar más soy yo. Y como siempre, siempre es la mamá la que más apegada a los hijos. (Mireya)

d) Las expectativas que les transmite la familia. La perspectiva que tiene la familia sobre el ingreso de las mujeres a capacitación para integrarse al mercado laboral, por lo general responde a los patrones de comportamiento establecidos tradicionalmente, es decir que es cuestionado. Los papás consideran que no es necesario que las mujeres tengan ingresos económicos ya que su pareja las va a mantener; los esposos o parejas, que es prioritaria la atención a la familia y el hogar, y los hijos e hijas consideran que su mamá ya no está en edad de capacitarse. Ante ello, las mujeres deben emprender procesos de negociación, que den certidumbre sobre su responsabilidad y habilidad para compaginar ambos entornos. Sin embargo, existen miembros cuya forma de pensar es difícil modificar, como en el caso de las suegras.

Empezó a decir: "no, es que después qué va a ser de mi hijo, quién le va a hacer de comer?”, y yo le decía:"tiene manitas, él puede hacerlo, ¿o qué le impide?”. "Entonces, ¿para qué quiere mujer?”... Decía mi suegra: “Tienes que pedir permiso". No, ellos salen y no piden permiso. Yo voy a hacer, estando bien, voy a seguir adelante y no va a haber quién me atore. “'Cómo no, tú familia!, ¿quién te las va a cuidar?”. ¡Yo! “¿Y vas a poder?”. Bendito Dios, que sí. (Margarita)

e) Romper los propios esquemas de pensamiento. De manera formal (en la escuela) o informal (familia y amigos), las mujeres han recibido una educación que ubica a las mujeres en el ámbito de lo privado, de ahí que su ingreso a la capacitación para entrar al mundo laboral implica un cambio en su propia ideología; sin embargo, en ningún 
caso implica una ruptura o una transformación total. Consideran que los hombres y mujeres son iguales y que deben participar de la misma manera en el ámbito laboral y familiar; sin embargo, ellas continúan con la doble jornada de trabajo. En todo caso, es en los hijos e hijas en quienes tratan de inculcar una forma de pensar y actuar respecto al género, más equitativa.

Muy contenta con lo que hago. Son mis deberes como mamá, como esposa, como mujer... Porque la mujer es como el pilar de su casa. La mujer tiene que estar en casa. No digo que no trabaje, sí, porque yo trabajo. Pero este, siempre dedicada al hogar, a los hijos, entre los dos educar. Pero que no olvide el hombre que él es el que tiene que arrimar más a casa, ¿verdad?, pero sin olvidarse de la esposa y de la educación de los hijos; hacer presencia de la paternidad. (Martha)

Sé que nos falta mucho a las mujeres, sí que nos falta mucho, ¿en qué forma? De que nosotras las mamás, nuestros hijos hombrecitos, enseñarlos a hacer todo: fregar, lavar, hacerlos igual lo que hace la mujer. (Margarita)

La conciliación entre la vida laboral y la familiar conlleva un conjunto de ajuestes que las mujeres deben realizar tanto de manera personal, como social. Como señala Silveira, "ellas deben optar o hacer filigranas que redundan en disponer de mayor tiempo para ellas mismas, se exponen a la sobreexigencia, se imponen restricciones a la convivencia con la multiplicidad de roles y con el registro de estar en falta, con la culpa que genera 'dejar los hijos o estar a medias con ellos'”. (Siveira, 2001: 472)

\section{Conclusión}

La investigación confirmó que la capacitación para el trabajo ha jugado un papel relevante en la transformación de la vida personal y laboral de las mujeres; sin embargo, el incremento de su formación e ingreso al mercado de trabajo no ha modificado sustancialmente la participación de los varones en las tareas del hogar, ni la forma de pensar respecto a las funciones sociales de las mujeres y los hombres.

No obstante la persistencia de la inequidad de género, es posible visualizar avances importantes. Las mujeres muestran conciencia de las desigualdades de género en el mercado laboral, tienden a demostrar que poseen tanta capacidad como los hombres para generar ingresos, aspiran a ser exitosas tanto en el ámbito doméstico como laboral y señalan el rumbo para incidir y modificar las condiciones de desarrollo de las nuevas generaciones, a través de la educación de sus hijos e hijas.

En afán de lograr la conciliación entre el mundo laboral y familiar, realizan una serie de ajustes entre los patrones de socialización tradicionales y sus aspiraciones, lo que repercute en la reconstrucción de sí mismas y sus proyectos de vida.

La capacitación para el trabajo significa una oportunidad para ampliar los horizontes de las mujeres, visualizar nuevas opciones de desarrollo personal y profesional, y establecer nuevas redes sociales. Además, la posibilidad de ejercer un oficio y generar ingresos les permite posicionarse a sí mismas como personas con mayor autonomía. Emplean con más libertad y autoridad el capital del que disponen, aunque en gran parte 
lo destinan para la familia más que en beneficio personal; reservan tiempos para cumplir con las actividades vinculadas a su habilitación, pero sólo en fines de semana; reclaman apoyo en las tareas y responsabilidades de la familia, pero no buscan ser sustituidas totalmente, y se ubican en la familia como proveedoras en el mismo nivel que los hombres, pero siguen considerando que ellos son un soporte fundamental en el hogar.

Elevar su autonomía no implica renunciar a sus responsabilidades reproductivas y familiares, sino que es parte de la conciliación que realizan con su vida familiar. Es decir, efectúan un conjunto de negociaciones en las cuales ellas distinguen qué ceden y qué reclaman, y es evidente que en este proceso, el cuidado de los hijos es prioritario. Esto implica que las mujeres asignan un valor elevado a los ámbitos doméstico y al privado, y consideran que no están en contradicción o enfrentamiento con la esfera pública, sino que son espacios diferenciados que es necesario concertar para salir adelante en la complicada sociedad actual.

Finalmente se puede decir, que es claro que la capacitación para el trabajo por sí sola no resolverá el problema de exclusión laboral y social de las mujeres, pero contibuye en abrir caminos para lograr su autonomía personal y económica.

\section{Referencias}

Aguilar, Teresa. 2008. El sistema sexo-género en los movimientos feministas. Amnis. https://amnis.revues.org/537 (13 de enero de 2014).

Cervantes, Irina. 2013. Los retos de los derechos de la mujer en Nayarit. En Soy Constitucional. Derechos fundamentales de los nayaritas, coordinado por Pedro Enríquez y José Madero. pp. 15-20. Nayarit: Poder Judicial del Estado de Nayarit.

Colín, Mónica, María Gallaga y Gloria Martínez. 2011. Trayectoria laboral de género: un estudio comparativo entre instituciones de educación superior públicas y privadas. Ponencia presentada en el VII Congreso Nacional de la Asociación Mexicana de Estudios del Trabajo. Mérida, Yucatán.

Fundación Mujeres. 2007. La conciliación de la vida laboral, familiar y personal: una estrategia que beneficia a las empresas. Badajoz: Proyecto MELKART, Iniciativa Comunitaria EQUAL.

Gamba, Susana. 2008. Diccionario de estudios de género y feminismo. Buenos Aires: Biblos.

Guest, Greg, Arwen Bunce y Laura Johnson. 2006. How many interviews are enough? An experiment with data saturation and variability. Field Methods, 18:59-82. DOI: https://doi.org/10.1177/1525822X05279903 Guzmán, Flérida, Hilda Rodríguez y Priscila González. 2008. Diagnóstico sobre las desigualdades de género en el Estado de Nayarit. Nayarit: INMUJERES.

Harding, Sara. 1987. Is there a feminist method? Feminism and Methodology. pp. 1-14. Bloomington: Indiana University Press.

Instituto Nacional de las Mujeres. 2003. Las mexicanas y el trabajo II. Ciudad de México: INMUJERES. Instituto Nacional de las Mujeres. 2004. El ABC de género en la administración pública. Ciudad de México: Instituto Nacional de las Mujeres, Programa de las Naciones Unidas para el Desarrollo (PNUD). Lamas, Martha. 1986. La antropología feminista y la categoría “género". Nueva antropología 8 (30): 173-198. Lamas, Martha. 1996. La perspectiva de género. La Tarea. Revista de educación y cultura. http://www. latarea.com.mx/articu/articu8/lamas8.htm (8 de junio de 2015).

Longo, María. 2009. Género y trayectorias laborales. Un análisis del entramado permanente de exclu- 
siones en el trabajo. Trayectorias. 11 (28): 118-141.

Luque, Martha. 2012. De lo privado y doméstico a lo público. Transformaciones de las relaciones de género en las mujeres de los sectores populares de Lima Metropolitana. Tesis de magíster en sociología, Universidad Nacional Mayor de San Marcos.

Martínez, Miguel. 2004. Ciencia y arte en metodología cualitativa. México: Trillas.

Miles, Matthew y Michael Huberman.1994. Qualitative data analysis. Londres: Sage Publications.

Pieck, Enrique. 2012. En el camino... formación para el trabajo e inclusión: ¿hacia dónde vamos? Ciudad de México: Universidad Iberoamericana, Institutos de Capacitación para el Trabajo.

Reyes, Rosa. 2005. La autonomía psicológica en mujeres profesionales: análisis de un caso. Otras miradas. http://www.redalyc.org/articulo.oa?id=18350101 (28 de enero de 2014).

Riquer, Florinda y Ana Tepichín. 2001. De la casa a la escuela, del trabajo a los quehaceres del hogar. En Los jóvenes y el trabajo: la educación frente a la exclusión social, coordinado por Enrique Pieck. pp. 493525. Ciudad de México: UIA, IMJUVE, UNICEF, CINTERFOR, CONALEP, RET.

Rocha, Tania y Cinthia Cruz. 2013. Barreras estructurales y subjetivas en la transición de roles de mujeres mexicanas y su malestar emocional. Acta Colombiana de Psicología, 16 (1): 123-135.

Sanz, Alexia. 2005. El método biográfico en la investigación social: potencialidades y limitaciones de las fuentes orales y los documentos personales. Asclepio, 57 (1): 99-115. DOI: https://doi.org/10.3989/ asclepio.2005.v57.i1.32

Sifuentes, Emma. 2002. Globalización, desarrollo local y género. En La condición actual de la mujer en Nayarit, coordinado por Fabiola González. pp. 133-145. Nayarit: Universidad Autónoma de Nayarit.

Silveira, Sara. 2001. La dimensión de género y sus implicaciones en la relación entre juventud, trabajo y formación. En Los jóvenes y el trabajo: la educación frente a la exclusión social, coordinado por Enrique Pieck. pp. 457-491. México: UIA, IMJUVE, UNICEF, CINTERFOR, CONALEP, RET. 